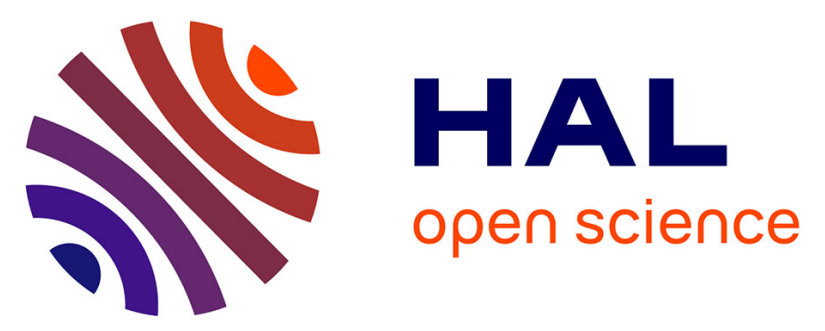

\title{
A polar $/ \pi$ model of interactions explains face-to-face stacked quinoid rings: a case study of the crystal of potassium hydrogen chloranilate dihydrate
}

Krešimir Molčanov, Jernej Stare, Biserka Kojić-Prodić, Claude Lecomte, Slimane Dahaoui, Christian Jelsch, Emmanuel Wenger, Ana Šantić, Bartosz Zarychta

\section{To cite this version:}

Krešimir Molčanov, Jernej Stare, Biserka Kojić-Prodić, Claude Lecomte, Slimane Dahaoui, et al.. A polar $/ \pi$ model of interactions explains face-to-face stacked quinoid rings: a case study of the crystal of potassium hydrogen chloranilate dihydrate. CrystEngComm, 2015, 17 (45), pp.8645-8656. 10.1039/C5CE01037C . hal-02112708

\section{HAL Id: hal-02112708 https://hal.science/hal-02112708}

Submitted on 26 Apr 2019

HAL is a multi-disciplinary open access archive for the deposit and dissemination of scientific research documents, whether they are published or not. The documents may come from teaching and research institutions in France or abroad, or from public or private research centers.
L'archive ouverte pluridisciplinaire HAL, est destinée au dépôt et à la diffusion de documents scientifiques de niveau recherche, publiés ou non, émanant des établissements d'enseignement et de recherche français ou étrangers, des laboratoires publics ou privés. 


\section{A polar $/ \pi$ model of interactions explains face-to-face stacked quinoid rings: a case study in the crystal of potassium hydrogen chloranilate dihydrate}

Krešimir Molčanov ${ }^{a}$, Jernej Stare ${ }^{b}$, Biserka Kojić-Prodića, Claude Lecomtec, Slimane Dahaoui ${ }^{c}$, Christian Jelsch $^{c *}$, Emmanuel Wenger ${ }^{c}$, Ana Šantića , Bartosz Zarychta ${ }^{d}$

a Rudjer Bošković Institute, Bijenička 54, HR-10000 Zagreb, Croatia.

E-mail: Kresimir.Molcanov@irb.hr

b National Institute of Chemistry, Hajdrihova 19, SI-1001 Ljubljana, Slovenia

c Cristallographie, Résonance Magnetique et Modélisations CNRS, UMR 7036, Institut Jean Barriol, CNRS and Université de Lorraine BP 70239, F54506

Vandoeuvre-les-Nancy CEDEX, France. E-mail: christian.jelsch@univ-lorraine.fr

d Faculty of Chemistry, Uniwersytet Opolski, Oleska 48, 45-052 Opole, Poland

The nature of interactions between face-to face alternate stacked quinoid rings with $\pi$-systems, observed with short inter-ring centroid...centroid distance is analyzed by experimental and theoretical methods. Charge density studies based on X-ray diffraction and DFT calculations, complemented by impedance spectroscopy were employed to define the electronic and structural characteristics of the quinoid rings responsible for their interactions within the crystal packing. The crystal packing is mainly stabilized by several salt bridges between the $\mathrm{K}^{+}$cation and the hydrogen chloranilate anions. The proximity and orientation of the stacked quinoid rings in parallel roto-inversion arrangement is mainly governed by non-covalent interactions of unusual strength. Their estimated energy, more than $10 \mathrm{kcal} \mathrm{mol}^{-1}$, as calculated by DFT methods, is comparable to mediumstrong hydrogen bonding. It is the result of the unique electronic structure of 2,5-dihydroxyquinonate monoanion, which exhibits alternating electron-rich and electron-poor regions. Thus, the nature of interactions between $\pi$-systems of quinoid rings can be described by a polar/ $\pi$ model. These interactions have a great potential in crystal engineering and may be employed in design of functional materials. 


\section{Introduction}

$\pi$-Interactions are quite common type of intermolecular interactions ${ }^{1-9}$ occurring between planar, conjugated $\pi$-electron systems and have already found their way into supramolecular chemistry textbooks ${ }^{10}$. However, their exact nature is still a matter of dispute ${ }^{5,11-13}$. $\pi$-Interactions are generally considered to be weak, with a typical energy lower than $1 \mathrm{kcal} / \mathrm{mol}^{10}$, but can be considerably enhanced by dipolar interactions ${ }^{5,9,14}$. Nevertheless, they have quite a potential in supramolecular chemistry ${ }^{15,16}$ and crystal engineering ${ }^{17}$.

An empirical model for aromatic $\pi$-stacking, developed by Hunter and Sanders ${ }^{1,6}$, states that attractive interactions prevail in parallel offset (Fig. 1a) and T-shaped (Fig. 1b) arrangement. Face-to-face eclipsed or alternated arrangements (Fig. 1c, d) are energetically unfavourable for aromatic hydrocarbons due to strong repulsion. However for arene-perfluoroarene dimers, Salonen et al. ${ }^{12}$ found by computation that the face-toface eclipsed $\mathrm{C}_{6} \mathrm{~F}_{6} \cdots \mathrm{C}_{6} \mathrm{H}_{6}$ dimer has a negative energy and is nearly as favourable as the parallel offset configuration. This is due to the complementary electrostatic potentials between the two molecules ${ }^{8}$. In the current study of a chloranilic acid salt, a new arrangement, parallel non-offset alternate is found.

Typically in aromatic $\pi$ - $\pi$ stacking, distances between ring centroids (and carbon atoms of contiguous rings) are greater than $3.6 \AA$ and greater than the sum of van der Waals radii for carbon, which is $3.5 \AA^{18}$ and offsets are larger than $1.7 \AA$ (about a half of the ring diameter). Such an arrangement observed in the crystal structures ${ }^{1}$ is in accordance with $a b$ initio calculations ${ }^{19-21}$ and suggests that aromatic $\pi$-interactions are weak electrostatic interactions between electron-rich $\pi$-electron cloud of one ring and electron-poor $\sigma$-electron skeleton of another ring (Fig. 2). In aromatic heterocyclic rings, but not in pure $(\mathrm{C}, \mathrm{H})$ compounds, $\mathrm{C}$... C contacts occur frequently in crystal packing as parallel offset $\pi$-stacking can be favorable from the electrostatic point of view ${ }^{12,22}$. While the study of $\pi$-interactions has been limited almost exclusively to aromatic rings, stacking of other types of planar conjugated rings is neglected.
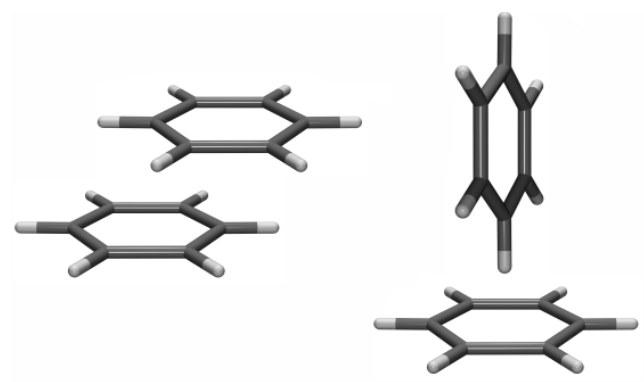

a)

b)

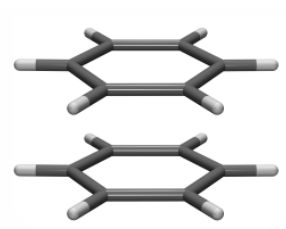

c)

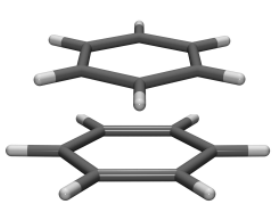

d)

Figure 1 Possible arrangements of stacked rings: a) parallel-offset and b) T-shaped are energetically favourable for aromatics; less common are parallel, face-to-face c) eclipsed and d) staggered. 


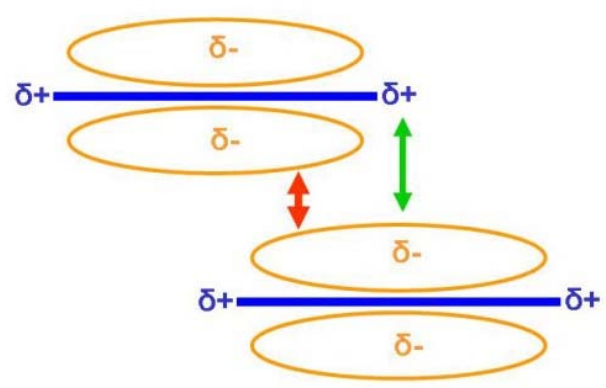

Figure 2 Interactions between two parallel, offset aromatic rings: green arrow indicates attraction between electron-poor $\sigma$ skeleton of one ring and electron-rich $\pi$ cloud of another, while the red arrow indicates repulsion of $\pi$ electron clouds (represented by ochre ovals).

Quinoid rings (Scheme 1) differ from aromatics due to distinguishable single and double bonds ${ }^{23}$. Their different electronic structures imply different types of intermolecular interactions. However, until recently no attempt has been made to study $\pi$-stacking of quinoid rings.

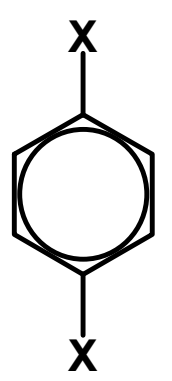

a)<smiles>[X]=c1ccc(=[X])cc1</smiles>

b) Scheme 1

A few years ago, we observed unusual face-to-face stacking in a few alkali salts of a quinoid compound, chloranilic acid (3,6-dichloro-2,5-dihydroxy-p-benzoquinone) ${ }^{24}$ (Scheme 2) which prompted us to further study the phenomenon. It turned out that alkali salts of various substituted 2,5-dihydroxyquinones tend to form face-to-face stacks ${ }^{25-28}$, both in eclipsed (Fig. 1c) ${ }^{28}$ and staggered (Fig. 1d) 24,26,27 $^{24 r a n g e m e n t s . ~ C e n t r o i d ~}$ and interplanar distances in such stacks are about $3.3 \AA(0.2 \AA$ shorter than the sum of van der Waals radii for carbon $^{18}$ ), indicating an unusually strong interaction. Preliminary quantum chemical calculations indicated that these interactions are comparable to hydrogen bonds, and are likely stronger than $10 \mathrm{kcal} \mathrm{mol}^{-}$ 1. Face-to-face alternate arrangement of quinoid rings in the stacks is such to allow close contact between electron-rich and electron-poor parts of the molecule: electron-rich $\pi$-bonds (double and delocalised) are sandwiched between electron-poor $\sigma$-bonds and vice versa (Fig. 3). Thus, $\sigma-\pi$ attractions are maximised, while $\pi-\pi$ repulsions are minimised.

Face-to-face alternate $\pi$-stacking has been described by Hunter $\&$ Sanders $^{1}$ (Fig. 1d); it is observed in crystal packing of 2,3,5,6-tetramethyl-1,4-benzoquinone (duroquinone) ${ }^{29}$ and several salts of 3,6-dibromo-2,5dihydroxyquinone (bromanilic acid) ${ }^{27}$. The rotation between two interacting parallel quinones is about $90^{\circ}$. 
However, such a simplified model, based exclusively on geometrical data (crystal structures measured with a resolution of $0.8 \AA$ ) leaves many questions opened. It does not provide an energy estimate of the interaction and says nothing on the possibility of localized charge transfers between two adjacent rings. Due to a close contact between the rings some degree of reciprocal charge transfer might be possible.

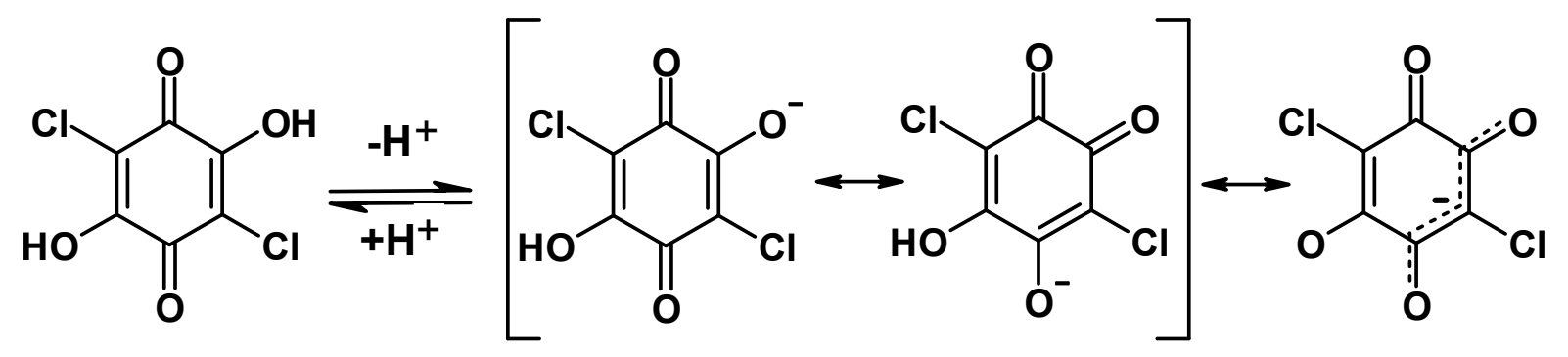

Scheme 2 Dissociation of chloranilic acid to the monoanion with resonance structures shown in brackets. The monoanion comprises single, double and delocalised C-C and C-O bonds.

Therefore, we decided for a detailed, in-depth study of interactions between face-to-face oriented quinoid rings by a combination of experimental X-ray charge-density, quantum chemical calculations ( $a b$ initio and periodic DFT), and measurement of electrical properties. As a very convenient model compound, we chose potassium hydrogen chloranilate dihydrate $\left(\mathrm{KHCA} \cdot 2 \mathrm{H}_{2} \mathrm{O} \text {, Scheme } 2\right)^{24}$. It is very stable and easy to prepare, grows in large well-developed crystals and comprises only light atoms, which do not introduce a problem of large X-ray absorption.

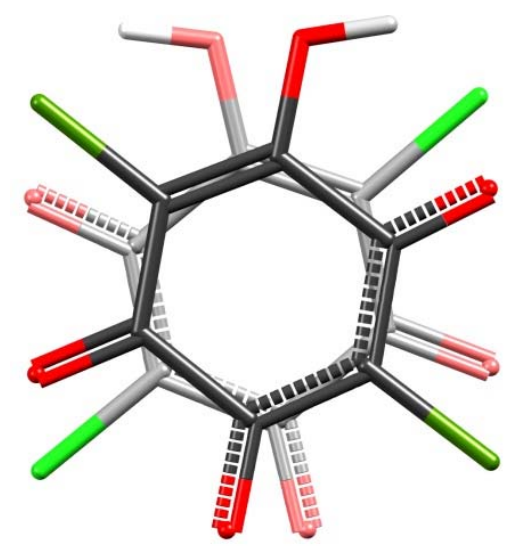

Figure 3 A simplified representation of face-to-face alternate (staggered) arrangement of hydrogen chloranilate anions related by a roto-inversion: electron-rich $\pi$-bonds (double and delocalised) are sandwiched between electron poor $\sigma$-bonds (single). Atoms are colour-coded: $\mathrm{C}$ black/dark gray, $\mathrm{O}$ red, $\mathrm{Cl}$ green, $\mathrm{H}$ light gray, and $\mathrm{K}$ violet. 


\section{Results and Discussion}

To gain insight into the nature of the face-to-face interaction, X-ray charge density analysis was assisted by DFT calculations utilizing periodic and isolated cluster models, revealing the role of non-bonding interactions and the energetics of stacking. Possible charge transfer between the rings was probed by impedance spectroscopy. A detailed study of crystal morphology is required for both, charge density determination (to correct absorption) and impedance spectroscopy (to apply electrical contacts at specific faces), but it also gives some insight into the dominant interactions in the crystal.

\section{Crystal morphology}

Crystals of KHCA $2 \mathrm{H}_{2} \mathrm{O}, P 2_{1} / c$, are needle-like, elongated in the direction of the $c$ axis, which is also the direction of ring stacking between equidistant (3.19 $\AA$ ) hydrogen chloranilate anions (Fig. 4). The largest and best developed forms are $\{100\}$ and the crystals are very thin in the direction of the $a$ axis. This implies that they grow rapidly in the direction [001], but very slowly in the direction [100]. Therefore, we can expect that the strongest intermolecular interactions are parallel to the $c$ axis and the weakest parallel to the $a$ axis. Indeed, the crystal packing comprises layers of cations, anions and water molecules linked together by cation '.anion interactions and hydrogen bonds; these layers are parallel to the plane (100) (Fig. 5, Table 1). They are linked by only two symmetry-independent hydrogen bonds (Table 1). Therefore, we should expect that the strong interaction in the direction [001] is mainly due to the $\mathrm{K}^{+}$/anion salt bridges linking two adjacent quinoid rings and secondarily to the face-to-face staggered arrangement of the rings. The potassium coordination is made of five chlorine and two oxygen atoms. On the other hand, there are two hydrogen bonds approximately perpendicular to the $c$ axis and one approximately parallel to it (Table 1).
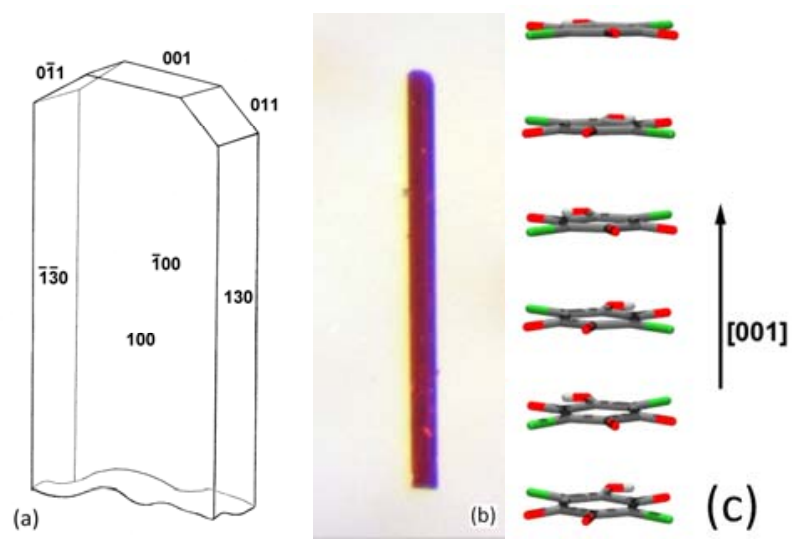

Figure 4 a) The crystal habit of $\mathbf{K H C A} \cdot \mathbf{2} \mathrm{H}_{2} \mathrm{O}$ with combinations of crystal forms; $\{011\}$ form is developed just exceptionally, b) photograph of the real sample (the crystal is $0.8 \mathrm{~mm}$ long), c) perspective view of a face-to-face arrangement of hydrogen chloranilate anions in KHCA.2 $\mathrm{H}_{2} \mathrm{O}$. 


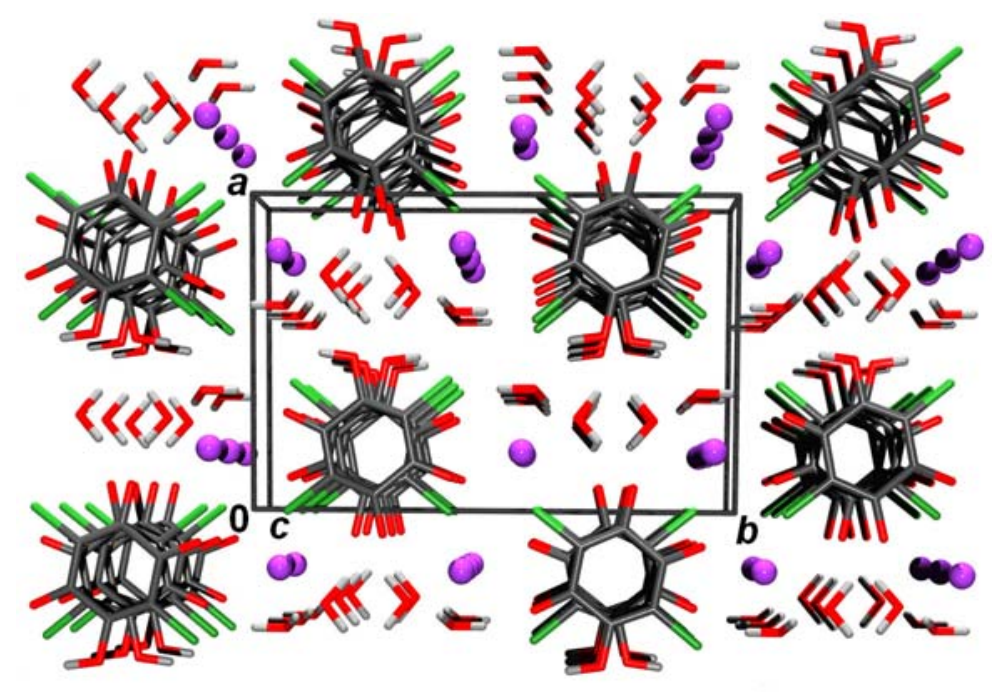

Figure 5 Crystal packing of $\mathbf{K H C A} \cdot 2 \mathbf{H}_{2} \mathbf{O}$ viewed approximately along the direction [001]. Potassium cations are shown as spheres of arbitrary radii.

Table 1 Geometric parameters of hydrogen bonds.

\begin{tabular}{|c|c|c|c|c|c|}
\hline & $\mathrm{d}(D-\mathrm{H})$ & $d(H \cdots A)$ & $\mathrm{d}(D \cdots A)$ & $D-\mathrm{H} \cdots A$ & Symm. operation on $A$ \\
\hline & / $\AA$ & $/ \AA ̊$ & $/ \AA ̊$ & $1^{\circ}$ & \\
\hline $\mathrm{O} 1-\mathrm{H} 1 \cdots \mathrm{O} 5$ & $0.967(2)$ & $1.827(2)$ & $2.613(3)$ & $136.4(6)$ & $-1+x, 3 / 2-y,-1 / 2+z$ \\
\hline O5-H5A $\cdots 06$ & $0.967(2)$ & $1.811(2)$ & $2.758(2)$ & $165.3(3)$ & $x, y, 1+z$ \\
\hline $\mathrm{O} 5-\mathrm{H} 5 \mathrm{~B} \cdots \mathrm{O} 2$ & $0.967(2)$ & $2.026(3)$ & $2.835(3)$ & $140.1(6)$ & $-x, 1 / 2+y, 1 / 2-z$ \\
\hline $\mathrm{O} 6-\mathrm{H} 6 \mathrm{~A} \cdots \mathrm{O} 3$ & $0.967(2)$ & $1.870(2)$ & $2.835(3)$ & $176.53(6)$ & $x, 3 / 2-y,-1 / 2+z$ \\
\hline $\mathrm{O} 6-\mathrm{H} 6 \mathrm{~B} \cdots \mathrm{O} 1$ & $0.967(2)$ & $1.993(2)$ & $2.954(3)$ & $173.2(1)$ & $1+x, y, z$ \\
\hline
\end{tabular}

Electronic structure of the hydrogen chloranilate anion based on X-ray charge density studies and DFT calculations

The experimental deformation electron density of the hydrogen chloranilate anion (Fig. 6a) is in a good agreement with the simplified structural formula (Scheme 2) derived from molecular geometry ${ }^{24}$. It is a very interesting system for studying covalent bonds since this relatively small anion comprises three types of C-C and C-O bonds: formally single, formally double and delocalised (bond order about 1.5). Table 2 gives the topological properties of all covalent bonds as calculated from multipolar refinement against experimental and theoretical intensities; the agreement between experiment and theory is very good. We note an overall larger charge density at CP for the experimental one. According to this topological analysis, C-C bonds can indeed be classified into these three types. Formally single bonds, $\mathrm{C} 1-\mathrm{C} 2, \mathrm{C} 4-\mathrm{C} 5$ and $\mathrm{C} 5-\mathrm{C} 6$, have the lowest electron density at the critical point and are the least elliptical. We note that both experimental and theoretical C5-C6 ellipticities are larger than 0.2; the C5-C6 bond is also shortened compared to typical C-C single bonds (1.51-1.54 $\AA$ ) in the Engh \& Huber dictionary ${ }^{30}$ and has considerably more electron density at the $\mathrm{CP}$ than typical single $\mathrm{C}-\mathrm{C}$ bonds, therefore it is more similar to delocalised 
bonds. The shortest formal single bond $\mathrm{C} 5-\mathrm{C} 6$ can be regarded as an elongated delocalised bond. Therefore, its bond order is quite greater than 1 . The formally double bond, $\mathrm{C} 6=\mathrm{C} 1$, has the largest electron density $\rho_{\mathrm{cp}}$ value, but is also longer than usual $\left(d=1.34 \AA^{31}\right)$ and its ellipticity is lower than the 0.45 value found in double bonds ${ }^{32}$; therefore its bond order is lower than two.

Table 2 Topology of C-C and C-O bonds derived from experimental and CRYSTALO9 computation electron-density after multipole refinement. Bond $\mathrm{C} 5-\mathrm{C} 6$ is listed as formally single, however, according to electron density it is more similar to delocalised bonds

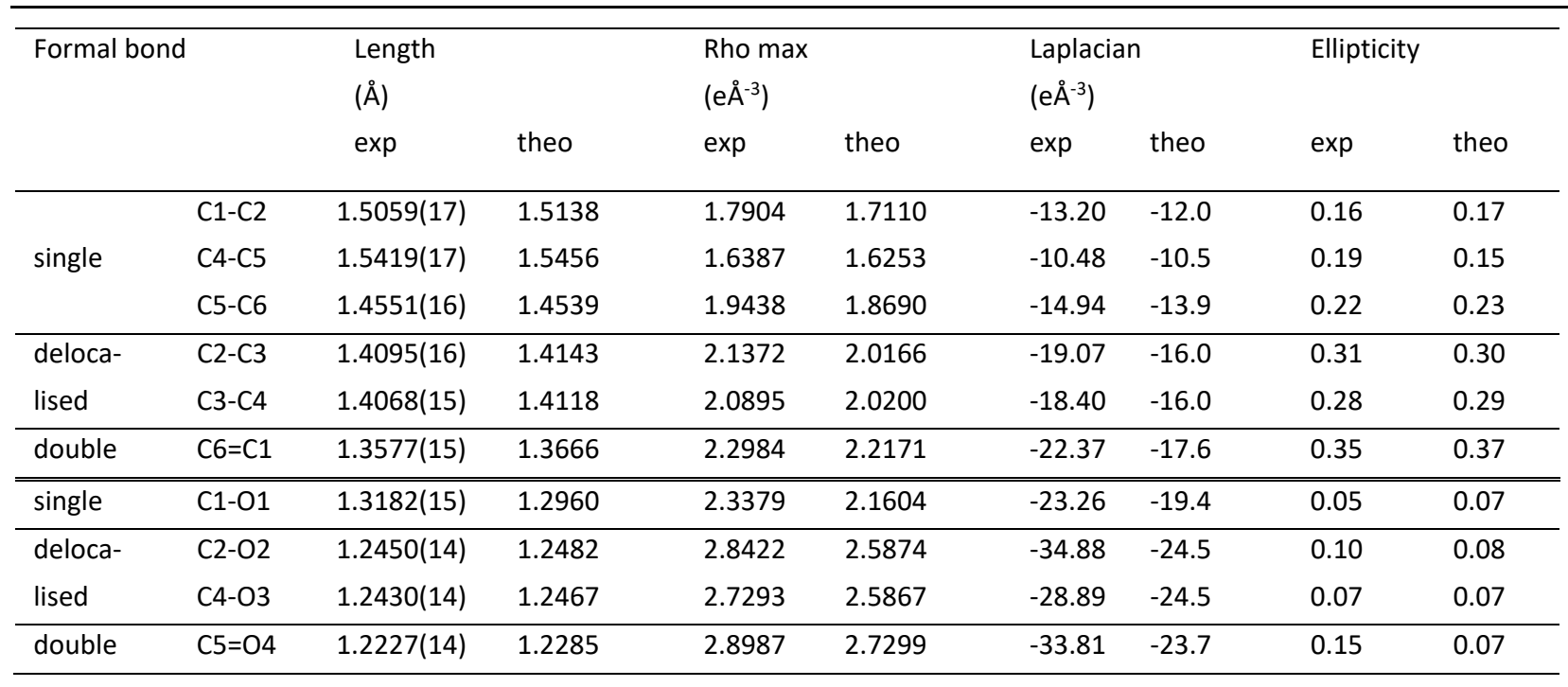

Topological properties of delocalized $\mathrm{C}-\mathrm{O}$ bonds (Table 2) are very close to those of a $\mathrm{C}=\mathrm{O}$ bond and the formally single bond, $\mathrm{C} 1-\mathrm{O} 1$, is $0.06 \AA$ shorter than similar $\mathrm{C}-\mathrm{O}$ bonds in hydroquinones ${ }^{33,34}$. It is also interesting to note that $\mathrm{C}-\mathrm{Cl}$ bonds have much lower $\rho_{\mathrm{cp}}$ electron density (in agreement with ref. ${ }^{35}$ ) than either $\mathrm{C}-\mathrm{O}$ or $\mathrm{C}-\mathrm{C}$ bonds $^{8}$, which can easily be noted in the deformation density map (Fig. 6a). Experimental and theoretical multipolar electrostatic potential (as calculated from ref. ${ }^{36}$ ) (Fig. $6 \mathrm{~b}$ and c) are also in excellent agreement with the simple delocalisation model (Scheme 2).

Most of the negative potential is located around $\mathrm{O}$ atoms as expected; the electrostatic potential around the chlorine atoms is less electronegative as already observed ${ }^{35}$. Atom $\mathrm{O} 4$, which is formally neutral is weaker attractor than $\mathrm{O} 2$ and $\mathrm{O} 3$. In the carbon skeleton, alternating electropositive and electronegative regions are observed: a negative electrostatic potential can be found in the regions of double and delocalised bonds, and positive about the single C-C bonds. This experimental picture supports our previous simple model (Scheme 2 and Fig. 3$)^{24}$ and is in a very good agreement with the quantum chemical calculations. The uneven distribution of electropositive and negative potential in the quinoid ring plays an important role in the stacking interactions, as shown below. 


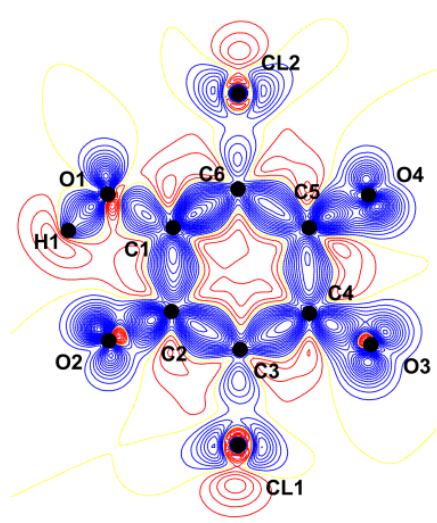

a)

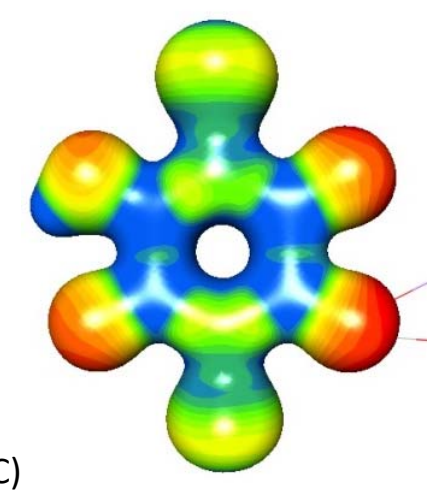

b)

Figure 6 a) Experimental deformation density of the hydrogen chloranilate anion. Positive density is blue and negative red; yellow dotted lines represent zero density. Contours are drawn for 0.05 e $\AA^{-3}$. b) Multipolar experimental and c) theoretical electrostatic potential mapped on electron density isosurface of 0.35 e $\AA^{-3}$. Colour scale -0.25 to +0.3 e $\AA^{-1}$.

\section{Anion $\cdots$ anion interactions as revealed by charge density analysis}

Since $\mathrm{KHCA} \cdot 2 \mathrm{H}_{2} \mathrm{O}$ is an ionic compound, the strongest and the most important interactions in the crystal packing are of ionic nature. Cation ' anion interactions therefore provide the "scaffolding" and more subtle details of the crystal packing are governed by weaker intermolecular interactions. We have studied quite a few alkali salts of chloranilic acid ${ }^{24-26}$ and its analogues ${ }^{27,28}$, which are sterically similar; however face-toface stacking occurs only in some of them. Dianions form offset, aromatic-like stacks ${ }^{24-27}$, while in the case of mono anions stacking is sometimes offset ${ }^{24}$ but, more commonly, face-to-face. While the steric effect of the cation certainly influences stacking, $\pi$-interactions between the rings should be regarded as the most important. However, since hydrogen chloranilate moieties are anions, strong repulsions between them should occur, compensated by cation $\cdots$ anion attractions ${ }^{37}$. The charge distribution in the chloranilate anion is such that most of the negative potential is located in the outer region of the anion oxygen atoms (Fig. 6b,c and Table S1 in the Supplement), which form close contacts with potassium cations (Fig. 5). Face-to-face arrangement of anions in the stacks then minimises repulsions while maximising any possible attractive interactions (dipolar and dispersive). This novel type of stacking and the fine-tuning of the interactions between quinoid rings was the main topic of this work. As we will show, it is the result of the peculiarly 
uneven distribution of electron density in the carbon ring leading to electro positive and electro negative regions.

The charge density analysis of these stacked anions in a face-to-face arrangement (Fig. 7a) reveals that the close contacts between the rings always involve electron-rich area of one ring and electron-poor area of another ring. Therefore, electrostatic repulsion is minimised, while at the same time both electrostatic (i.e. attractions between electron-rich and electron-poor regions) and dipolar attractions are maximised. Topological analysis of electron density revealed three $(3,-1)$ critical points between carbon atoms of contiguous rings with electron density maximum exceeding 0.05 e $\AA^{-3}$ (Table 4, Fig. 8) and four other (3,1) critical points between the substituent $\mathrm{O}$ and $\mathrm{Cl}$ atoms similar to those observed in the noncentrosymmetric phase of the TTF-CA charge transfer complex ${ }^{8}$ (Table 4, Fig. 8). Bond paths are observed linking the atoms. Also a $(3,+3)$ critical point is found between the rings, indicating a centre of a cage. This is in a good agreement with our previous simplified model (Fig. 3), and also indicates that the interaction is mostly polar excluding electron transfer between the rings; this is also confirmed by electrical conductivity measurements using a single crystal sample (Fig. 11 and Experimental section). The electrical conductivity along $c$ axis is $2.1 \times 10^{-11}(\Omega \mathrm{cm})^{-1}$ at room temperature indicating insulating characteristics of the sample. One more question to be answered would be whether the total interaction between the rings is attractive or repulsive; it can be answered by inspecting the electron density between the two rings. Between carbon skeletons, electron density reaches at least $0.05 \mathrm{e}^{-3}$ (Fig. $7 \mathrm{~b}$ ), similar to other noncovalent attractive interactions ${ }^{8}$. It should also be noted that the electron density between substituted atoms on the ring goes effectively down to lower values than in the inner region in Fig. 7b compared to Fig. 7a.

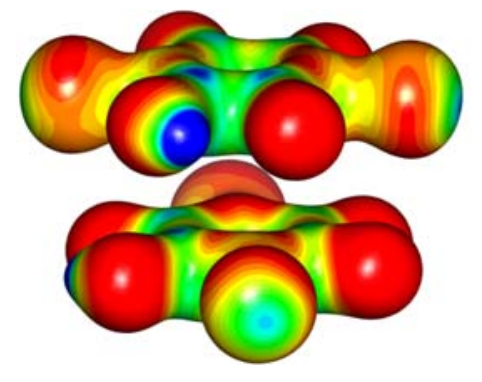

a)

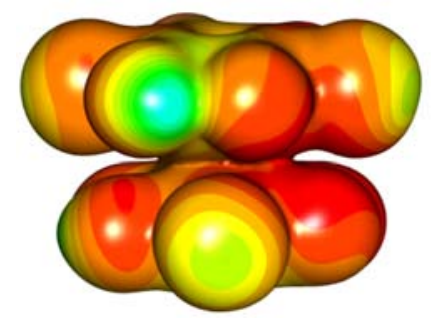

b)

Figure 7 Multipolar experimental electrostatic potential of a pair of contiguous hydrogen chloranilate rings a) mapped on an isosurface of 0.35 e $\AA^{-3}$ showing interactions between electron-rich (red and orange) and electronpoor (blue, green) regions, b) mapped on an isosurface of 0.05 e $\AA^{-3}$ showing electron density between carbon skeletons. Scale: a) $-0.35-+0.35$; b) $-0.6-+0.2$ e $\AA^{-1}$

The electrostatic energy between two anions using the experimental multipolar atom model is repulsive $\left(+65.7 \mathrm{kcal} \mathrm{mol}^{-1}\right)$. However when only the six carbon of the ring are considered, the electrostatic interaction energy $E_{\text {elec }}=-6.4 \mathrm{kcal} \mathrm{mol}^{-1}$ is indeed attractive (see Supporting Information, Table S2). Therefore, it is indeed a polar $\sigma-\pi$ interaction, consistent with the simplified model (Fig. 3). 
Table 4 Experimentally determined and calculated saddle $(3,-1)$ critical points within a pair of hydrogen chloranilate anions.

\begin{tabular}{|c|c|c|c|c|c|c|}
\hline$A \cdots B$ & $\rho$ tot & & Lapl & $\mathrm{n} \rho$ & type & Symm. operation on $A$ \\
\hline & $\exp$ & theo & $\exp$ & theo & & \\
\hline $\mathrm{C} 3 \cdots \mathrm{C} 5$ & 0.0560 & 0.0488 & 0.59 & 0.55 & $(3,-1)$ & $x, 3 / 2-y,-1 / 2+z$ \\
\hline $\mathrm{C} 3 \cdots \mathrm{C} 5$ & 0.0527 & & 0.56 & & $(3,-1)$ & $x, 3 / 2-y, 1 / 2+z$ \\
\hline $\mathrm{C} 6 \cdots \mathrm{C} 2$ & p.0550 & 0.0514 & 0.58 & 0.57 & $(3,-1)$ & $x, 3 / 2-y,-1 / 2+z$ \\
\hline $\begin{array}{l}\mathrm{Cl} 1 \cdots \mathrm{O} \\
4\end{array}$ & 0.0431 & 0.0447 & 0.61 & 0.61 & $(3,-1)$ & $x, 3 / 2-y, 1 / 2+z$ \\
\hline $\begin{array}{l}\mathrm{Cl} 2 \cdots \mathrm{O} \\
2\end{array}$ & 0.0435 & 0.0439 & 0.59 & 0.60 & $(3,-1)$ & $x, 3 / 2-y,-1 / 2+z$ \\
\hline $\begin{array}{l}\mathrm{Cl} 1 \cdots \mathrm{O} \\
4\end{array}$ & 0.0400 & 0.0391 & 0.49 & 0.51 & $(3,-1)$ & $x, 3 / 2-y,-1 / 2+z$ \\
\hline $\begin{array}{l}\mathrm{Cl} 2 \cdots \mathrm{O} \\
2\end{array}$ & 0.0368 & 0.0405 & 0.57 & 0.56 & $(3,-1)$ & $x, 3 / 2-y, 1 / 2+z$ \\
\hline $\mathrm{C} 1 \cdots \mathrm{C} 3$ & 0.0266 & 0.0212 & 0.29 & 0.26 & $(3,3)$ & $x, 3 / 2-y,-1 / 2+z$ \\
\hline
\end{tabular}

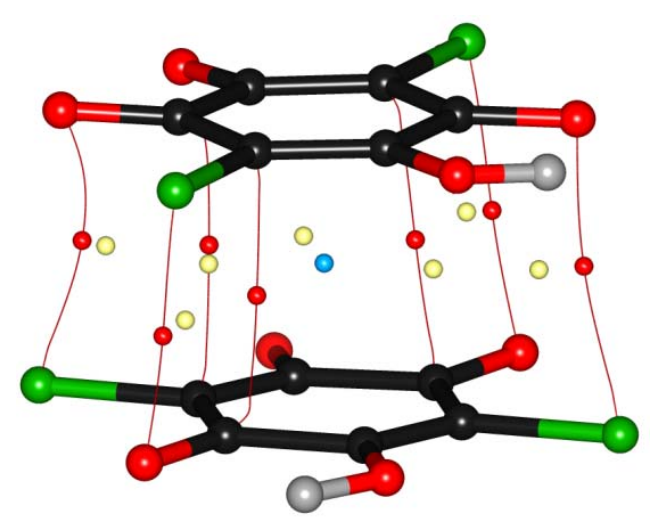

Figure 8 Critical points in a $\pi$-stack. $(3,-1)$ CPs and their bond paths are shown in red, $(3,+1)$ ring CPs are in yellow and $(3,+3)$ cage $C P$ is in blue.

Table 5 Geometric parameters defining $\pi \cdots \pi$ stacking arrangements between two adjacent rings $\mathrm{C} 1 \rightarrow \mathrm{C} 6 \cdots \mathrm{C} 1 \rightarrow \mathrm{C} 6$.

\begin{tabular}{ll}
\hline Plane distance $(\AA)$ & $3.1746(2)$ \\
$\mathrm{Cg} \cdots \mathrm{Cg}$ ' distance $(\AA)$ & $3.176(3)$ \\
$\mathrm{Cg} \cdots \mathrm{Cg}^{\prime}$ offset $(\AA)$ & 0.087 \\
$\alpha$ angle between two plane rings. & 0.00 \\
6 angle between $\mathrm{Cg} \cdots \mathrm{Cg}^{\prime}$ line and normal to & 1.58 \\
planes $\left({ }^{\circ}\right)$ & \\
dihedral angle $\mathrm{C6}-\mathrm{Cg}-\mathrm{Cg}^{\prime}-\mathrm{C}^{\prime} 2^{\prime}\left({ }^{\circ}\right)$ & 101 \\
\hline
\end{tabular}

Symmetry operator on $\mathrm{Cg}^{\prime}$ and $\mathrm{O3}^{\prime}: x, 3 / 2-y,-1 / 2+z$ 


\section{Stacking of hydrogen chloranilate anions in view of DFT calculations}

In order to elucidate the nature of interactions governing the stacking of the $\mathrm{HCA}^{-}$quinoid rings and their proximity, we performed DFT calculations on dimers, by (approximately) excluding or including dispersion interactions. Due to constraints imposed by the size of the system, the DFT approach appears to be the method of choice. While clearly advantageous by fully supporting periodicity, many 'traditional' DFT methods such as B3LYP or PBE, are notorious for their lack of dispersion and, consequently, poor description of non-bonding interactions. Fortunately, recent development of DFT methodologies facilitates the inclusion of dispersion corrections in various forms. In such a way, DFT methods offer good compromise between accuracy and affordability even when non-covalent interactions represent a crucial factor for the structure of crystalline solids.

In this work, we employed a comparative treatment of a selected model, by using DFT without and with dispersion corrections; the role of non-covalent interactions may be qualitatively deduced by comparing the results of the two. For periodic DFT, as implemented in VASP, the Grimme DFT-D2 correction can be simply toggled on or off. For gas phase calculations by Gaussian, we used two distinct functionals, namely B3LYP and M06-2X, of which the former lacks dispersion while the latter reportedly accounts for it. We used a similar comparative strategy in our previous investigations of the stacked quinoid rings, yielding reasonable results ${ }^{28}$.

PERIODIC CALCULATIONS. Starting from the experimental crystal structure data, we performed full optimization of the system, including atomic positions and unit cell parameters. Table 6 lists the optimized unit cell parameters obtained by either using or omitting the DFT-D2 dispersion correction.

Table 6 Optimized unit cell parameters and volume of $\mathrm{KHCA} \cdot 2 \mathrm{H}_{2} \mathrm{O}$ calculated without and with the DFT-D2 dispersion corrections of the PBE functional. Experimental values are listed as reference.

\begin{tabular}{llll}
\hline & $\begin{array}{l}\text { dispersion } \\
\text { OFF }\end{array}$ & $\begin{array}{l}\text { dispersion } \\
\text { ON }\end{array}$ & experimental \\
\hline$a[\AA]$ & 10.051 & 9.889 & $9.986(9)$ \\
$b[\AA]$ & 15.338 & 15.308 & $15.210(16)$ \\
$c[\AA]]$ & 6.647 & 6.213 & $6.350(5)$ \\
$b\left[^{\circ}\right]$ & 100.04 & 98.86 & $99.09(3)$ \\
$V\left[\AA^{3}\right]$ & 1009. & 929. & $952 .(2)$ \\
\hline
\end{tabular}

There is a steady trend of shrinking of the unit cell on including the dispersion correction, which is a clear evidence that non-covalent, non-directional attractive forces play a significant role in the structure of the system. The dispersion-included approach yields clearly better agreement with the experimental structure for the above listed parameters. This is in agreement with findings published in our previous work ${ }^{28}$. By far, the largest difference between the dispersion-included and dispersion-omitted method is in the value of the unit cell constant $c$. Inclusion of dispersion reduces the value of $c$ by no less than $0.434 \AA$, and the dispersioncorrected methodology provides significantly better agreement with the experiment. Since the HCA' rings stack along the cell vector $c$ and the stacking distance is equal to half the length of parameter $c$, this provides 
strong evidence that, among the structural motifs of the present system, the van der Waals interaction component between the rings most significantly influences the stacking proximity.

Gas PHASE CAlCUlations. We further investigated the role of non-bonding interactions on a stacked dimer model of two $\mathrm{HCA}^{-}$rings. Fig. 9 displays the interaction energy of two rings as a function of their torsional alignment, calculated by the dispersion-deficient B3LYP and the dispersion-enhanced M06-2X functional.

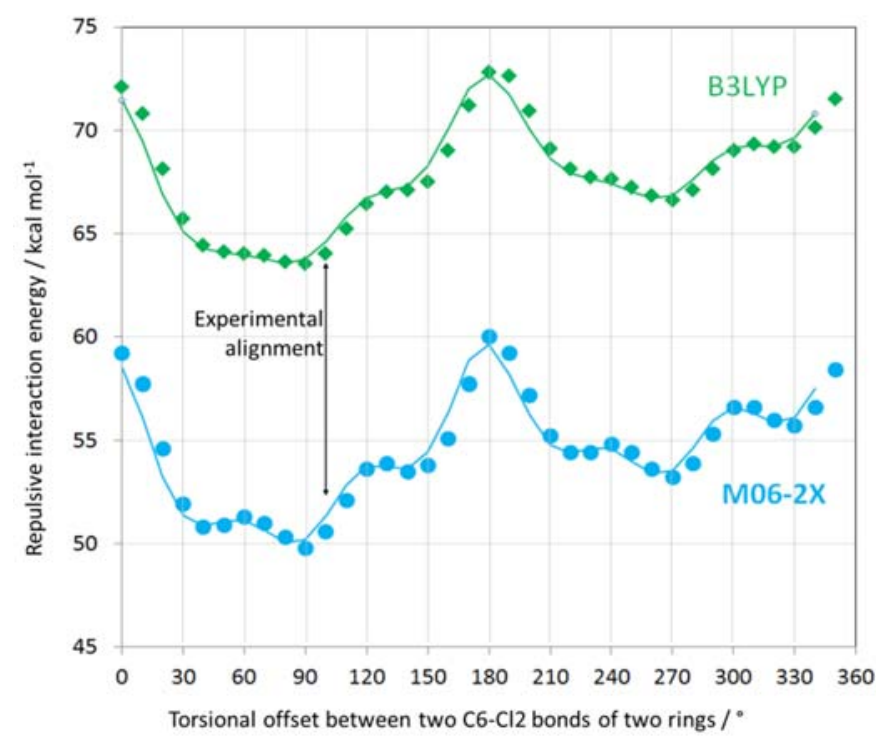

Figure 9 Counterpoise-corrected pairwise interaction energy of a pair of stacked HCA rings, computed with the B3LYP and M06-2X functionals and the $6-311++G(2 d, 2 p)$ basis set, as function of their torsional alignment (see Fig. 10).

Both profiles exhibit significant repulsion originating from the fact that each ring bears a negative charge. The profiles are nearly identical, spanning a range of about $10 \mathrm{kcal} \mathrm{mol}^{-1}$ from lowest to highest repulsion. Remarkably, the experimental torsional alignment of about $101^{\circ}$ (expressed as torsional offset between the C6-Cl2 bonds, see Fig. 10) is very close to the global minimum of both profiles. This confirms that the alignment of the rings follows the tendency of minimizing electrostatic repulsion and also supports the aforementioned view that the overlap between the electron-rich and electron-poor domains of the neighbouring rings provides substantial stabilization. The $\sim 13 \mathrm{kcal} \mathrm{mol}^{-1}$ shift between the B3LYP and M06$2 \mathrm{X}$ profile demonstrates significantly lower repulsion derived from the latter functional. We believe that a significant part of this difference can be attributed to non-covalent interaction between the rings, since B3LYP is known to be deficient in this aspect while M06-2X appears to be including the dispersion component more properly. Although quantitative determination of the $\pi$-interaction component is beyond the reach of the present study, it can be safely assumed that its amount is substantial. The computed difference in interaction energy between M06 and B3YLP is nearly twice as large as found in our previous study of a system of stacked dianion quinoid rings $\left(\sim 7 \mathrm{kcal} \mathrm{mol}^{-1}\right)^{28}$, which is likely to be attributed to the closer stacking distance (by more than $0.3 \AA$ ) in the present case. 


\section{Conclusions}

This paper presents a detailed study of an unusual type of face-to-face stacking, as a contribution to the development of a more general theory of stacking of planar $\pi$-systems. Substituted quinones have revealed quite often face-to-face arranged quinoid rings ${ }^{24-27}$ with separation distance significantly shorter than the sum of carbon $\cdots$ carbon van der Waals radii. Their stacking motifs and geometrical parameters are different to those observed for aromatic rings. The recent theoretical papers ${ }^{11,13,19,38-40}$, which discuss the nature of interactions between aromatic rings, came to conclusion that ' $\pi$-stacking' and ' $\pi \cdots \pi$-interactions' do not accurately describe the forces responsible for association of aromatics. A more plausible explanation is that the aromatic $\pi$-interactions are weak electrostatic interactions between electron-rich $\pi$-electron cloud of one ring and electron-poor $\sigma$-electron skeleton of another ring, and may be enhanced by the presence of antiparallel molecular dipoles. From these papers is emerging a more general concept that is valid not only for aromatics but also for other $\pi$-systems, including quinoid rings.

In the system studied, potassium hydrogen chloranilate dihydrate, quinoid rings in the stacks are equidistant, with interplanar separation of 3.176(3) $\AA$ and a negligible offset of only $0.087 \AA$ (compared to more than $1.6 \AA$ in aromatic stacks).

Two adjacent $\mathrm{C}_{6}$-rings appear alternate, with a rotation of $11^{\circ}$. While ionic interactions are dominant in the crystal packing, face-to-face alternate stacking is directed by more subtle polar $\sigma-\pi$ interactions between the quinoid rings' delocalised systems. The energy of interaction was estimated by quantum chemical calculations to be at least $-10 \mathrm{kcal} \mathrm{mol}^{-1}$, which is by one order of a magnitude stronger than better-known aromatic $\pi$-interactions.

Our findings on the nature of interactions between $\pi$-systems of quinoid rings are in line with the most recent papers $^{8,13,19,38-40}$ which consider polar $\sigma-\pi$ model instead of specialised $\pi \cdots \pi$ interactions. The polar $\sigma-\pi$ model is realistic and can be applied to all molecules having $\pi$-systems (not only for aromatics).

\section{Experimental}

\section{X-ray diffraction and multipolar refinement}

The crystals were prepared as reported previously ${ }^{24}$. Morphology of the crystals was studied using a Unicam optical goniometer. X-ray diffraction measurements were performed on a Bruker D8 Venture diffractometer at $100 \mathrm{~K}$. Full Ewald sphere was measured with the maximum resolution of $0.50 \AA$. Program package Bruker Apex ${ }^{41}$ was used for data reduction and analytical absorption correction. The multiple integrated reflections were averaged for the space group $P 2_{1} / C$ using SORTAV ${ }^{42}$ adapted to area detector data. Spherical-atom model was refined using SHELXL-97 ${ }^{43}$; atomic coordinates were taken from the room-temperature structure $^{24}$.

For multipolar refinement (vs. $F^{2}$ ) the program package MoPro ${ }^{44}$ was used. Anisotropic parameters for hydrogen atoms were calculated by the SHADE server ${ }^{45}$ and imported into the multipolar model (and constrained during the refinement); O-H distances were restrained to 0.967(2) $\AA$. Since the two water molecules have higher thermal motion, constraints had to be applied on the charge density. The four water $\mathrm{H}$ 's were set to be chemically equivalent as well as their two $\mathrm{O}$ atoms. Potassium cation was refined as a monopole. Wenger ${ }^{46}$ in his $\mathrm{PhD}$ thesis showed that high resolution scale factors are accurate and constant over the resolution range only when a pixel hybrid detector is used contrary to CCD or CMOS data. Then as these data are CMOS data, a polynomial scale factor as a function of $s=\sin \theta / \lambda$ : 
$I_{\mathrm{obs}}=I_{\text {calc }} * k\left(1+\alpha_{2} s^{2}+\alpha_{3} s^{3}\right)$

Such a correction which is available in Mopro $^{43}$ leads to significant reduction of residual electron density around the potassium atom.

Geometry, charge-density and electrostatic properties calculations were performed by MoPro ${ }^{44}$; molecular graphic were prepared using MoProViewer ${ }^{47}$ and ORTEP-3 ${ }^{48}$. Crystallographic and refinement data for KHCA $2 \mathrm{H}_{2} \mathrm{O}$ are shown in Table 7 ; additional details on refinement are deposited as Supporting Information. 
Table 7 Crystallographic, data collection and experimental charge-density refinement details.

\begin{tabular}{|c|c|}
\hline Compound & $\mathrm{KHCA} \cdot 2 \mathrm{H}_{2} \mathrm{O}$ \\
\hline Empirical formula & $\mathrm{C}_{6} \mathrm{H}_{5} \mathrm{Cl}_{2} \mathrm{KO}_{6}$ \\
\hline Formula wt. / $\mathrm{g} \mathrm{mol}^{-1}$ & 283.096 \\
\hline Crystal dimensions / mm & $0.415 \times 0.052 \times 0.031$ \\
\hline Space group & $P 2_{1} / c$ \\
\hline$a / \AA$ & $9.986(9)$ \\
\hline$b / \AA$ & $15.210(16)$ \\
\hline$c / \AA$ & $6.350(5)$ \\
\hline$\alpha /^{\circ}$ & 90 \\
\hline$B /{ }^{\circ}$ & $99.09(3)$ \\
\hline$v /{ }^{\circ}$ & 90 \\
\hline$z$ & 4 \\
\hline$V / \AA^{3}$ & $952 .(2)$ \\
\hline$D_{\text {calc }} / \mathrm{g} \mathrm{cm}^{-3}$ & 1.975 \\
\hline$\mu / \mathrm{mm}^{-1}$ & 1.123 \\
\hline $\sin \theta / \lambda$ & 1.0182 \\
\hline$T / \mathrm{K}$ & $100(2)$ \\
\hline Radiation wavelength & $0.71073(\mathrm{MoK \alpha})$ \\
\hline Diffractometer type & Bruker D8 Venture \\
\hline Range of $h, k, l$ & $\begin{array}{l}-20<h<20 \\
-30<k<30 \\
-12<l<12\end{array}$ \\
\hline Reflections collected & 179543 \\
\hline Independent reflections & 7923 \\
\hline $\begin{array}{l}\text { Observed reflections } \\
(I \geq 2 \sigma)\end{array}$ & 5688 \\
\hline Absorption correction & Analytical \\
\hline$T_{\min }, T_{\max }$ & $0.725,0.972$ \\
\hline Weighting scheme & $w=1 /\left[0.13^{*} \backslash s^{2}\left(F_{o}^{2}\right)\right]$ \\
\hline$R_{\text {int }}$ & 0.0432 \\
\hline$R(F)$ & 0.0132 \\
\hline$R_{w}\left(F^{2}\right)$ & 0.0263 \\
\hline Goodness of fit & 0.554 \\
\hline $\mathrm{H}$ atom treatment & $\begin{array}{l}\text { restrained, } \\
\text { anisotropic }\end{array}$ \\
\hline No. of parameters & 397 \\
\hline No. of restraints & 5 \\
\hline$\Delta \rho_{\max }, \Delta \rho_{\min }\left(\mathrm{e} \AA^{-3}\right)$ & $0.672 ;-0.467$ \\
\hline
\end{tabular}




\section{Quantum chemical calculations}

Both periodic and isolated models were considered. Periodic calculations were performed by the program package VASP v. $5.2^{49}$, including full geometry optimization of the crystal structure (atomic positions and cell parameters). The PBE functional ${ }^{50}$ was used together with a planewave basis set with a cutoff of $500 \mathrm{eV}$ and the Projector Augmented Wave atomic pseudopotentials ${ }^{51}$. A sufficiently dense $3 \times 2 \times 5 k$-point mesh was employed. Optimization was performed both without and with the addition of the Grimme DFT-D2 dispersion correction ${ }^{52}$ to the original PBE functional. Symmetry constraints of the $P 2{ }_{1} / C$ space group were followed during optimization.

The gas phase calculations were performed by the Gaussian 09 program suite ${ }^{53}$. The model consisted of a pair of stacked hydrogen chloranilate rings extracted from the experimental crystal structure. By rotating the rings along the line passing through their centres perpendicularly to the plane of the rings (Fig. 10), their pair interaction energy was calculated for each configuration by both the B3LYP ${ }^{54}$ and M06-2X ${ }^{55}$ functional in conjunction with the $6-311++\mathrm{G}(2 \mathrm{~d}, 2 \mathrm{p})$ basis set. The interaction energies were corrected for basis set superposition error by using the counterpoise method. During the scan the internal structure (bond lengths and valence angles) of the chloranilate anions was fixed to the experimental geometry. For practical reasons the rings were made planar, which was the only (very subtle) modification of the experimental data. Additionally, a comparison of the interaction energy between the M06-2X functional and the perturbation post-HF method MP2 ${ }^{56}$ was also made, using a pair of stacked rings aligned in the same way as in the experimental structure.

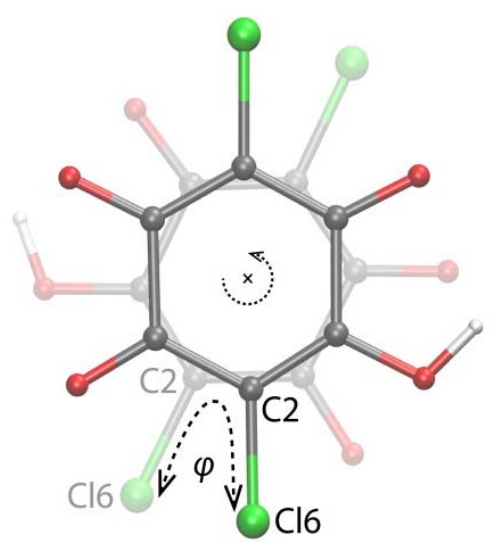

Figure 10 Model for the calculation of orientation-dependent interaction energy of a stacked pair of hydrogen chloranilate ions (the displayed case corresponds to $\varphi=30^{\circ}$ ).

\section{Theoretical charge density}

Periodic quantum mechanical calculation using CRYSTAL09 577

were performed at the crystal geometry observed experimentally and, using this as a starting geometry, optimization was performed with density functional theory (DFT) method ${ }^{58}$ and with the $B 3 L Y P$ hybrid functional ${ }^{59}$ using 6-31G(d,p) basis set ${ }^{60}$ 
For the potassium atom, the triple-zeta valence with polarization basis set developed by Peintinger et al. ${ }^{61}$ was used.

Upon convergence on energy ( $\Delta E \sim 10^{-6}$ hartree), the periodic wave function based on the optimized geometry was obtained. The coordinates of hydrogen atoms were relaxed, but the unit cell was kept fixed. The index generation scheme proposed by Le Page \& Gabe ${ }^{62}$ was applied to generate Miller indices up to $s=1.2 \AA^{-1}$ reciprocal resolutions. The option XFAC of the CRYSTAL09 program was then used to generate a set of theoretical structure factors from the computed electron density and using set of prepared indices. The theoretical charge density was refined vs. all the generated structure factors amplitudes.

\section{Theoretical multipolar refinement}

The multipole refinement based on the theoretical structure factors $F(h k l)$ was performed using all reflections up to resolution $d=0.42 \AA$. The atomic positions were fixed to the values obtained from the geometry relaxation, the scale factor was fixed to unity and atomic thermal motion parameters were set to zero. The valence and multipole populations, and kappa coefficients were refined together with block-diagonal option in several cycles until convergence. Neither restraints nor constraints were imposed to the electron density distribution of the atoms, except for the $\mathrm{H}$ atoms, for which the parameters were restrained to a value of 1.16(1).

\section{Measurement of electical conductivity}

Electrical conductivity of the single crystal sample along the crystallographic $c$ axis was measured by impedance spectroscopy (Novocontrol Alpha-N dielectric analyser) in the frequency range $0.01 \mathrm{~Hz}-1 \mathrm{MHz}$ at room temperature. For the electrical contacts, silver paint electrodes (rectangular, $0.417 \mathrm{~mm} \times 0.083 \mathrm{~mm}$ ) were deposited on the opposite surfaces (i.e. $\{001\}$ faces) of the crystal. The impedance spectrum was analysed by equivalent circuit modelling using the complex nonlinear least-squares fitting procedure (ZView software).

The complex impedance plot of the single crystal exhibits an arc at high values of impedance indicating low electrical conductivity (Fig. 11).

The experimental impedance data can be approximated by the equivalent circuit consisting of a parallel combination of resistor and capacitor. The parameters of the equivalent circuit, i.e. electrical resistance $(R)$ and capacitance $(C)$, obtained by the complex non-linear least squares fitting are listed in Figure 11. From the values of electrical resistance $(R)$ and electrode dimensions ( $A$ is the electrode area and $d$ is the sample thickness) DC conductivity is calculated according to relation: $\sigma_{\mathrm{DC}}=d /(A \cdot R)$. The electrical conductivity of the single crystal sample at room temperature equals to $2.1 \times 10^{-11}(\Omega \mathrm{cm})^{-1}$. 


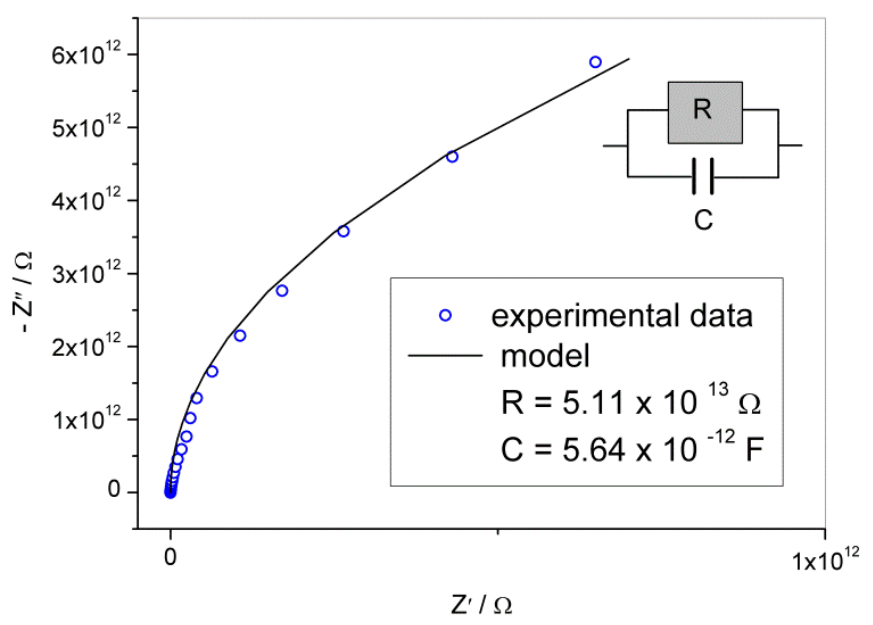

Figure 11 Complex impedance plot and the corresponding equivalent circuit.

\section{Acknowledgements}

The financing from the Ministry of Science, Education and Sports of the Republic of Croatia (Slovenian-Croatian bilateral collaboration grant for years 2012-2013), French-Croatian bilateral - Hubert Curien grant for years 2015-2016, the Croatian Academy of Sciences and Arts (for 2014) and Slovenian Research Agency (grant no. P1-0012) is gratefully acknowledged. K. M. is grateful to a joint RBI/French Embassy scholarship for a visit to Nancy. Diffraction measurements were performed at Institut Jean Barriol X-ray diffraction facility.

\section{References}

1 C. A. Hunter \& J. K. M. Sanders, J. Am. Chem. Soc., 1990, 112, 5525-5534.

2 C. A. Hunter, Angew. Chem., Int. Ed., 1993, 32, 1584-1586.

3 C. A. Hunter, Chem. Soc. Rev., 1994, 23, 101-109.

4 M. L. Głowka, D. Martynowski \& K. Kozłovska, J. Mol. Struct., 1999, 474, 81-89.

5 C. Janiak, J. Chem. Soc., Dalton Trans., 2000, 3885-3896.

6 C. A. Hunter, K. R. Lawson, J. Perkins \& C. J. Urch, J. Chem. Soc., Perkin Trans. 2, 2001, 651-659.

7 E. A. Mayer, R. K. Castellano \& F. Diedrich, Angew. Chem. Int. Ed. 2003, 42, 1210-1250.

8 P. García, S. Dahaoui, C. Katan, M. Souhassou \& C. Lecomte, Faraday Discuss., 2007, 135, $217-$ 235.

9 S. E. Wheeler, Acc. Chem. Res., 2013, 46, 1029-1038.

10 J. W. Steed \& J. L. Atwood, Supramolecular Chemistry, $2^{\text {nd }}$ Ed., J. Wiley \& Sons, Chichester, U.K., 2009.

11 S. Grimme, Angew. Chem. Int. Ed. 2008, 47, 3430-3434.

12 L. M. Salonen, M. Ellermann \& F. Diedrich, Angew. Chem., Int Ed., 2011, 50, 4808-4842.

13 C. R. Martinez \& B. L. Iverson, Chem. Sci., 2012, 3, 2191-2201.

14 K. Molčanov \& B. Kojić-Prodić, CrystEngComm, 2010, 12, 952-939.

15 M. W. Hosseini, Acc. Chem. Res., 2005, 38, 313-323. 
16 F. J. M. Hoeben, P. Jonkheim, E. W. Meijer \& A. P. H. J. Schenning, Chem. Rev., 2005, 105, 14911546.

17 J. K. Klosterman, Y. Yamauchi \& M. Fujita, Chem. Soc. Rev., 2009, 38, 1714-1725.

18 B. Cordero, V. Gómez, A. E. Platero-Prats, M. Revés, J. Echeverría, E. Cremades, F. Barragán \& S. Alvares, Dalton Trans., 2008, 2832-2838.

19 E. C. Lee, D. Kim, P. Jurečka, P. Tarakeshwar, P. Hobza \& K. S. Kim, J. Phys. Chem. A, 2007, 111, 3446-3457.

20 M. O. Sinnokrot \& D. C. Sherril, J. Phys. Chem. A, 2006, 110, 10656-10668.

21 Y. C. Park \& J. S. Lee, J. Phys. Chem. A, 2006, 110, 5091-5095.

22 C. Jelsch, K. Ejsmont \& L. Huder, IUCrJ, 2014, 1, 119-128.

23 S. Hünig, Pure \& Appl. Chem., 1990, 62, 395-406.

24 K. Molčanov, B. Kojić-Prodić \& A. Meden, CrystEngComm, 2009 11, 1407-1415.

25 K. Molčanov, B. Kojić-Prodić \& A. Meden, Croat. Chem. Acta, 2009, 82, 387-396.

26 K. Molčanov, B. Kojić-Prodić \& I. Sabljić, CrystEng Comm, 2011, 13, 4211-4217.

27 K. Molčanov \& B. Kojić-Prodić, Acta Cryst. B, 2012, B68, 57-65.

28 K. Molčanov, B. Kojić-Prodić, D. Babić \& J. Stare, CrystEngComm, 2013, 15, 135-143.

29 R. Shukla \& R. Rathore, Synthesis, 2008, 3769-3774.

30 R. A. Engh \& R. Huber, Acta Cryst. 1991, A47, 392-400.

31 M. A. Fox \& J. K. Whitesell, Organische Chemie: Grundlagen, Mechanismen, Bioorganische Anwendungen. Springer. 1995.

32 D. Stalke, Chem. Eur. J., 2011, 17, 9264-9278.

33 K. Molčanov, B. Kojić-Prodić \& M. Roboz, Acta Cryst. B., 2006, B62, 1051-1060.

34 K. Molčanov, B. Kojić-Prodić, D. Babić, D. Žilić \& B. Rakvin, CrystEngComm, 2011, 13, 5170-5178.

35 T. T. T. Bui, S. Dahaoui, C. Lecomte, G. R. Desiraju \& E. Espinosa, Angew. Chem., Int. Ed., 2009,

48, 3838-3841.

36 N. Ghermani, C. Lecomte \& N. Bouhmaida, Z. Naturforsch. Teil A, 1983, 48, 91-98.

37 C. Lecomte, E. Espinosa \& C. F. Matta, IUCrJ, 2015, 2, 161-163.

38 P. Munshi \& T. N. Guru Row, Crystallogr. Rev., 2005, 11, 199-241.

39 A. Coskun, J. Spruell, G. Barin, A. Fahrenbach, R. Forgan, M. Colvin, R. Carmieli, D. Benítez, E.

Tkatchouk, D. Friedman, W. A. Goddard III \& J. Stoddart, J. Am. Chem. Soc. 2011, 133, 45384547.

40 J. Spruell, A. Coskun, D. Friedman, R. Forgan, A. Sarjeant, A. Trabolsi, A. Fahrenbach, G. Barin, W. Paxton \& S. Dey, Nature Chem. 2010, 2, 870-879.

41 APEX2, SADABS and SAINT. Bruker AXS Inc., Madison, Wisconsin, USA, 2010.

42 R. H. Blessing, Crystallogr. Rev., 1987, 1, 3-58.

43 G. M. Sheldrick, Acta Crystallogr. 2008, A64, 112-122.

44 C. Jelsch, B. Guillot, A. Lagoutte \& C. Lecomte. J. Appl. Cryst., 2005, 38, 38-54.

45 A. Ф. Madsen, J. Appl. Cryst., 2006, 39, 757-758.

46 a)E. Wenger, PhD thesis, Université de Lorraine, May 2015; b) E. Wenger, P. Allé, S. Dahaoui,

D. Schaniel \& C. Lecomte, Acta Cryst. B, to be published. 
47 B. Guillot. Acta Cryst A., 2012, A68, s204.

48 L. J. Farrugia, J. Appl. Cryst., 1997, 30, 565.

49 (a) G. Kresse \& J. Hafner, Phys. Rev. B, 1993, 47, 558-561.; (b) G. Kresse \& J. Hafner, Phys. Rev.

B, 1994, 49, 14251-14269.; (c) G. Kresse \& J. Furthmüller, Comput. Mat. Sci., 1996, 6, 15-60.;

(d) G. Kresse \& J. Furthmüller, Phys. Rev. B, 1996, 54, 11169-11186.

50 J. P. Perdew, K. Burke \& M. Ernzerhof, Phys. Rev. Lett., 1996, 77, 3865-3868.

51 (a) P. E. Blochl, Phys. Rev. B, 1994, 50, 17953-17979.; (b) G. Kresse \& D. Joubert, Phys. Rev. B, 1999, 59, 1758-1775.

52 S. Grimme, J. Comp. Chem., 2006, 27, 1787-1799.

53 M. J. Frisch, G. W. Trucks, H. B. Schlegel, G. E. Scuseria, M. A. Robb, J. R. Cheeseman, G. Scalmani, V. Barone, B. Mennucci, G. A. Petersson, H. Nakatsuji, M. Caricato, X. Li, H. P. Hratchian, A. F. Izmaylov, J. Bloino, G. Zheng, J. L. Sonnenberg, M. Hada, M. Ehara, K. Toyota, R. Fukuda, J. Hasegawa, M. Ishida, T. Nakajima, Y. Honda, O. Kitao, H. Nakai, T. Vreven, J. A. Montgomery, J. E. Peralta, F. Ogliaro, M. Bearpark, J. J. Heyd, E. Brothers, K. N. Kudin, V. N. Staroverov, R. Kobayashi, J. Normand, K. Raghavachari, A. Rendell, J. C. Burant, S. S. Iyengar, J. Tomasi, M. Cossi, N. Rega, J. M. Millam, M. Klene, J. E. Knox, J. B. Cross, V. Bakken, C. Adamo, J. Jaramillo, R. Gomperts, R. E. Stratmann, O. Yazyev, A. J. Austin, R. Cammi, C. Pomelli, J. W. Ochterski, R. L. Martin, K. Morokuma, V. G. Zakrzewski, G. A. Voth, P. Salvador, J. J. Dannenberg, S. Dapprich, A. D. Daniels, O. Farkas, J. B. Foresman, J. V. Ortiz, J. Cioslowski, D. J. Fox, Gaussian 09, Revision A.02; Gaussian, Inc.: Wallingford CT, 2009.

54 (a) A. D. Becke, J. Chem. Phys., 1993, 98, 5648-52.; (b) C. Lee, W. Yang, \& R. G. Parr, Phys. Rev. $B, 1988,37,785-89$.

55 Y. Zhao \& D. G. Truhlar, Theor. Chem. Acc., 2008, 120, 215-41.

56 (a) C. Møller \& M. S. Plesset, Phys. Rev., 1934, 46, 618-622.; (b) M. Head-Gordon, J. A. Pople, \& M. J. Frisch, Chem. Phys. Lett., 1988, 153, 503-06.

57 R. Dovesi, V. R. Saunders, C. Roetti, R. Orlando, C. M. Zocovich-Wilson, F. Pascale, B. Civalleri, K. Doll, N. M. Harrison, I. J. Bush, P. D'Arco. \& M. Llunell, CRYSTAL-09 User's Manual, University of Turin, Turin, 2009.

58 P. Hohenberg \& W. Kohn, Phys. Rev., 1964, B136, 864-871.

59 C. Lee, W. Yang \& R. G. Parr, Phys. Rev. B, 1988, 37, 785-789.

60 P. C. Hariharan \& J. A. Pople, Theor. Chim. Acta, 1973, 28, 213-222.

61 M. F. Peintinger, D. Vilela Oliveira \& T. Bredow. J. Comp. Chem., 2012, 34, 451-459.

62 Y. Le Page \& E. J. Gabe, J. Appl. Cryst., 1979, 12, 464-466. 